\title{
THE COX, INGERSOLL AND ROSS EXTENDED MODEL
}

\author{
Wojciech Szatzschneider* \\ Escuela de Actuaría, Universidad Anáhuac del Norte
}

(Received 6 november 2001, accepted 28 january 2002)

\begin{abstract}
This paper presents a simple construction of the extended Cox, Ingersoll \& Ross model (ECIR.) for term structure of interest rate, and a simple way of pricing general interest rate derivatives with this model. To price zero-cupon bonds, we calculate the Laplace transform of functionals of an "elementary process", which is taken as a squared Bessel process. This approach takes advantage of martingales and the Girsanov's theorem providing a presentation of results in a "user friendly" form. Furthermore, we propose and apply a method for the calibration of the one factor ECIR model using data of bonds prices.
\end{abstract}

\section{Resumen}

Este trabajo presenta una construcción simple del modelo extendido de Cox, Ingersoll y Ross para la estructura de plazos de la tasa de interés, así como una forma simple de valuar productos derivados generales de tasa de interés con este modelo. Para valuar bonos cupón cero, se calcula la transformada de Laplace de funcionales de un proceso "elemental", el cual es tomado como un proceso cuadrático de Bessel. Este enfoque aprovecha las ventajas de la propiedad de martingala y del teorema de Girsanov permitiendo la presentación de resultados en una forma amigable. Además, se propone y aplica un método elemental para la calibración del modelo de un factor ECIR mediante registros históricos de los precios de bonos.

JEL classification: $E 43$

Keywords: Term structure of interest rates, Bessel processes, Girsanov theorem, Time transformation, Bonds and option pricing

* Escuela de Actuaría, Universidad Anáhuac del Norte, Avenida Lomas Anáhuac s/n, Col. Lomas Anáhuac, Huixquilucan, 52786 Edo. de México, Telephone: 52(55)5627-0210, E-mail: wojciech@anahuac.mx

The author is very grateful to the anonymous referees for their comments. 


\section{Introduction}

In this section we provide a short presentation of both the Gaussian and squared Gaussian models for term structure of interest rates. These models correspond to the first and second orders of complexity of random variables measurable with respect to $\sigma\{W(s), s \geq 0\}$, see Yor (1992), and might be seen as the simplest models for a short rate. Following Rogers (1995), the extended Vasicek (EV) model in the risk-neutral world is defined by

$$
\mathrm{d} r(t)=\widetilde{\sigma}_{t} \mathrm{~d} W(t)+\left(\widetilde{\alpha}_{t}-\widetilde{\beta}_{t} r(t)\right) \mathrm{d} t
$$

and the extended Cox, Ingersoll \& Ross model (ECIR) by

$$
\mathrm{d} r(t)=\widetilde{\sigma}_{t} \sqrt{r(t)} \mathrm{d} W(t)+\left(\tilde{\alpha}_{t}-\tilde{\beta}_{t} r(t)\right) \mathrm{d} t
$$

Here, $\widetilde{\alpha}, \tilde{\beta}$, and $\widetilde{\sigma}$ are positive and locally bounded (bounded in any finite interval) functions.

From now on, we will use a different parametrization of ECIR coming out naturally from our approach. These models were considered by Hull \& White (1990) who generalized models constructed by Vasicek (1977), and Cox, Ingersoll \& Ross (1985). It is easy to see that both models are affine in the short rate in the sense that

$$
P(t, T)=A(t, T) e^{-r_{t} B(t, T)},
$$

where $P(t, T)$ is the price at time $t$ of the bond maturing at $T$. On the other hand, the condition

$$
\frac{\widetilde{\alpha}_{t}}{\widetilde{\sigma}_{t}^{2}}=\text { constant }
$$

called constant dimension condition, was considered in the case of ECIR by Jamshidian, of. Rogers (1995). Precisely, this same condition will appear in our approach although it can be extended to the general case.

Even in the original CIR model with constant parameters, many different generalized $\chi^{2}$ distributions that appear in various papers and textbooks (including those on pricing simple options) may produce an aversion to put this model into practice. Our goal is to simplify things and show that pricing problems in ECIR are relatively easy. Even the statistical procedures to determine underlying parameters (functions of time) can be performed smoothly. In the following subsection, we will provide the description of our approach.

\subsection{Description of a basic model}

We define a $\delta$-dimensional squared Bessel process-BESQ ${ }^{\delta}, X_{t}$, as the unique strong solution of the following stochastic differential equation:

$$
\mathrm{d} X_{t}=2 \sqrt{X_{t}} \mathrm{~d} W(t)+\delta \mathrm{d} t
$$


Let us recall the Shiga \& Watanabe theorem, sometimes called Pythagoras theorem: $X_{t}^{\delta_{1}} \oplus X_{t}^{\delta_{2}} \equiv X_{t}^{\delta_{1}+\delta_{2}}$, where $\oplus$ stands for an independent sum. This theorem will play an important role in our modeling and pricing.

\subsection{ECIR Model redefined}

We will show in section 1.3 that, when taking $X_{t}=B E S Q^{\delta}$ ( $\delta$-dimensional squared Bessel process) as a basic model, and applying a change of measure, we will have the process $Y_{t}$ that satisfies

$$
\mathrm{d} Y_{t}=2 \sqrt{Y_{t}} \mathrm{~d} W(t)+\left(2 \beta_{t} Y_{t}+\delta\right) \mathrm{d} t, \quad Y_{0}=X_{0}
$$

Here, $\beta(t)<0$ is a locally bounded and a $C^{1}$ function.

We will later drop the above condition, and allow for discontinuities. At this moment, we focus into a model with this restriction in order to make our arguments easier. Moreover, we multiply the process $Y_{t}$ by a deterministic function $\sigma_{t}>0, \sigma_{t}$ locally bounded. If the function $\sigma_{t} \in C^{1}$ (we do not strictly require this condition), then $r(t)=\sigma_{t} Y_{t}$ satisfies

$$
\mathrm{d} r(t)=2 \sqrt{\sigma_{t} r(t)} \mathrm{d} W(t)+\left\{\left[2 \beta_{t}+\frac{\sigma_{t}^{\prime}}{\sigma_{t}}\right] r(t)+\delta \sigma_{t}\right\} \mathrm{d} t
$$

and condition (3) is satisfied. Now, there exist unique functions $\beta, \sigma$ and a constant $\delta$ that match the future market specifications; that is, the prices of bonds and their volatilities. For details, we once more refer to Rogers, who takes the original CIR as a base and "perturbs" the process through:

(i) deterministic $C^{2}$ time change, and

(ii) multiplication by a deterministic $C^{1}$ function.

The class of processes obtained by his method is the same as in our approach. This time-change technique is also used by Maghsoodi (1996). We will use the time change but only to find the corresponding probability laws needed in option pricing, and not to construct the model.

\subsection{Change of measure}

Let $X_{t}$ be a $\mathrm{BESQ}^{\delta}$ process, and consider the continuous exponential local martingale

$$
\bar{Z}_{t}=\mathcal{E}\left(\int_{0} \beta_{s} \sqrt{X_{s}} \mathrm{~d} W_{s}\right)_{t}=\exp \left\{\frac{1}{2} \int_{0}^{t} \beta_{s} \mathrm{~d} M_{(s)}-\frac{1}{2} \int_{0}^{t} \beta_{s}^{2} X_{s} \mathrm{~d} s\right\},
$$

where $M(s)=X_{s}-\delta s$. It readily results that

$$
\bar{Z}_{t}=\exp \left\{\frac{1}{2}\left[\beta_{t} X_{t}-\beta_{0} X_{0}-\delta \int_{0}^{t} \beta_{s} \mathrm{~d} s-\int_{0}^{t}\left(\beta_{s}^{2}+\beta_{s}^{\prime}\right) X_{s} \mathrm{~d} s\right]\right\} .
$$


If $\beta_{s}^{2}+\beta_{s}^{\prime} \geq 0$, the local martingale $\bar{Z}_{t}$ is bounded $\left(X_{s} \geq 0\right.$ and $\left.\beta_{t}<0\right)$, and therefore is a martingale. If not, we can use a slightly more elaborated argument, called Novikov criterion for small intervals. Therefore, in any case $\bar{Z}_{t}$ is a martingale, and the change of drift via the Girsanov's theorem is justified.

\section{Pricing bonds in ECIR Model}

We will now show how to price zero-coupon bonds in the ECIR model. At time zero (to simplify the notation), we have

$$
\begin{aligned}
P(0, u) & =E\left[\exp \left(-\int_{0}^{u} r(s) \mathrm{d} s\right)\right] \\
& =E\left[\exp \left(-\int_{0}^{u} Y_{s} \sigma_{s} \mathrm{~d} s\right)\right] \\
& =E\left[\exp \left(-\int_{0}^{u} X_{s} \sigma_{s} \mathrm{~d} s\right) \bar{Z}(u)\right] \\
& =\exp \left[\frac{1}{2}\left(-\beta_{0} X_{0}-\delta \int_{0}^{u} \beta_{s} \mathrm{~d} s\right)\right] \\
& \times E\left\{e^{\left.\frac{1}{2}\left[\beta_{u} X_{u}-\int_{0}^{u} X_{s}\left(\beta_{s}^{2}+\beta_{s}^{\prime}+2 \sigma_{s}\right) \mathrm{d} s\right]\right\}}\right.
\end{aligned}
$$

We look for $F_{u}(s)=F(s)$ such that $F^{2}(s)+F^{\prime}(s)=\beta_{s}^{2}+\beta_{s}^{\prime}+2 \sigma_{s}$, for $s \in[0, u)$, $F(u)=\beta_{u}$. Such an $F$ exists and is of finite variation. Moreover, it is easy to solve this equation by numerical methods. Now, we can apply the approach from section 1.3 with $F$ playing the role of $\beta$, and the expectation in (9), completed with the corresponding deterministic function

$$
\exp \left\{\frac{1}{2}\left(-F(0) X_{0}-\delta \int_{0}^{t} F(s) \mathrm{d} s\right)\right\}
$$

which is equal to 1 . In this part, the most suitable approach to the Riccati equation, that defines $F$, is via the corresponding Sturm-Liouville equation. Writing $F(s)=\varphi^{\prime}(s) / \varphi(s), s \in[0, u), \varphi(0)=1$, we get

$$
\frac{\varphi^{\prime \prime}(s)}{\varphi(s)}=h(s)=\beta_{s}^{2}+\beta_{s}^{\prime}+2 \sigma_{s} \text { in }[0, u), \quad \frac{\varphi^{-}(u)}{\varphi(u)}=\beta_{u},
$$

where $\varphi^{-}$is the left-hand side derivative. It is easy to see that

$$
\frac{\varphi^{\prime}(s)}{\varphi(s)}<\beta_{s} \text { for } s \in[0, u) \text {. }
$$

The exponential martingale that corresponds to $F$ will be called $Z$, as follows,

$$
Z_{t}=\mathcal{E}\left(\int_{0} F(s) \sqrt{X(s)} d W_{s}\right)_{t}
$$


( $Z$ corresponds to $F$ in the same way as $\bar{Z}$ to $\beta$ ). Whenever necessary, we will stress the dependence of $Z$ on $u$ and write $Z_{u}$. Note that the equation

$$
\frac{\varphi^{\prime \prime}}{\varphi}=c^{2}
$$

were $c$ is a positive constant $c$, can be solved easily as we will show later, so if $\beta_{s}^{2}+\beta_{s}^{\prime}+2 \sigma_{s}$ is constant, then the prices of bonds can be found explicitly.

The solution of the equation $\varphi^{\prime \prime}(s) / \varphi(s)=c^{2}$ in $[0, u)$ is clearly $A e^{c s}+$ $B e^{-c s}$ with conditions $A+B=1$ (because of $\varphi(0)=1$ ) and

$$
c\left(\frac{A e^{c u}-B e^{-c u}}{A e^{c u}+B e^{-c u}}\right)=\beta_{u} .
$$

Finally, in the general case, we obtain after elementary computations

$$
P(0, u)=\exp \frac{1}{2}\left\{\left[\ln \varphi_{u}(u)-\int_{0}^{u} \beta_{s} \mathrm{~d} s\right] \delta+x\left(-\beta_{0}+\varphi_{u}^{\prime}(0)\right)\right\},
$$

where $x=r_{0} / \sigma_{0}$. We set here $\varphi_{u}$ to stress the dependence on $u$ of the solution $\varphi(s)$ in $[0, u]$. In the same way, $F=F_{u}$. Using the comparison criterion for diffusion processes, or directly from (10), we obtain the intuitively obvious fact that the prices of bonds are decreasing (deterministic) functions of the short rate, and the explicit form for $A$ and $B$ in the affine representation:

$$
P(0, u)=A(0, u) e^{-B(0, u) r(0)} .
$$

Our main result may be written easily as:

$$
\mathrm{E}_{x}^{\delta}\left[\exp \left(-\frac{1}{2} \int_{0}^{u} X_{s} \mu(\mathrm{d} s)\right)\right]=\exp \left\{\frac{1}{2}\left[\varphi_{\mu}^{\prime}(0) x+\delta \ln \varphi_{\mu}(u)\right]\right\}
$$

where $\mu$ is the measure with density $h(s)$ in $[0, u)$, and the atom $-\beta(u)$ at $u$, $x=X_{0}=r(0) / \sigma_{0}, \varphi=\varphi_{\mu}$. In the case of $h>0$, this is precisely CameronMartin-Pitman-Yor's formula, cf. Revuz \& Yor (1999). It is well known that to price bonds in the ECIR model, one has to solve a Riccati equation, $c f$. Rogers (1995), but the approach presented here is via the corresponding SturmLiouville equation.

Now, we will show how easy our approach results in the case of piecewiseconstant parameters $\beta_{s}$ and $\sigma_{s}$ (this because of our parametrization of ECIR). This case was considered by Schlögl \& Schlögl (1997) using a different approach involving many different $\chi^{2}$ distributions. We will show how to price bonds $P(0, t)$ in the case of $\beta_{s}=\beta_{i}$, and $\sigma_{s}=\sigma_{i}$ for $s \in\left[t_{i}, t_{i+1}\right), i=0,1, \ldots, n-$ $1, t_{n}=t, \beta_{t}=\beta_{n}$. As before, we assume, $\beta_{i}<0, \sigma_{i}>0$. Consider

$$
\int_{0}^{t} X(s) d \beta_{s}=\sum_{i=1}^{n}\left(\beta_{i}-\beta_{i-1}\right) X_{t_{i}}
$$


and

$$
\begin{aligned}
P(0, t)= & \mathrm{E}\left\{\operatorname { e x p } \left\{\frac { 1 } { 2 } \left(\beta_{t} X_{t}-\beta_{0} X_{0}-\delta \int_{0}^{t} \beta_{s} \mathrm{~d} s\right.\right.\right. \\
& \left.\left.\left.\left.-\sum_{i=1}^{n}\left[\left(\beta_{i-1}^{2}+2 \sigma_{i-1}\right) \int_{t_{i-1}}^{t_{i}} X(s) \mathrm{d} s\right]-\sum_{i=1}^{n}\left(\beta_{i}-\beta_{i-1}\right) X_{t_{i}}\right\}\right)\right\}\right\} .
\end{aligned}
$$

As expected, $\beta_{n}=\beta_{t}$ are canceled (changing $\beta$ in one ending point does not affect the price of a bond). Note that we have chosen the right continuous version of $\beta$. It is clear that we solve the problem of bond pricing if we find the function $\varphi(s), s \in[0, t]$, such that:

i) $\varphi(s)$ is continuous,

ii) $\varphi^{\prime \prime}(s) / \varphi(s)=\beta_{i-1}^{2}+2 \sigma_{i-1}$ in $\left(t_{i-1}, t_{i}\right)$,

iii) $\left(\varphi^{+}\left(t_{i}\right)-\varphi^{-}\left(t_{i}\right)\right) / \varphi\left(t_{i}\right)=\beta_{i}-\beta_{i-1}$

iv) $\varphi^{-}\left(t_{n}\right) / \varphi\left(t_{n}\right)=\beta_{n-1}$,

v) $\varphi(0)=1$.

Here, $i=1,2, \ldots, n-1$, and $\varphi^{+}\left(\varphi^{-}\right)$stands for right (left) hand side derivative, respectively. Because the general solution of $\varphi^{\prime \prime}(s) / \varphi(s)=c_{i-1}^{2}$ is $A_{i-1} e^{c_{i-1} s}+$ $B_{i-1} e^{-c_{i-1} s}$, the problem is reduced to the solution of $2 n$ linear equations for $A_{i-1}, B_{i-1}, i=1, \ldots, n$. Recalling our general formula for $P(0, t)$ we only need to find $A_{0}$ and $A_{n-1}$ because $B_{0}=1-A_{0}$, and $B_{n-1}$ is recovered from the condition $i v$ ) since

$$
\frac{\varphi^{-}\left(t_{n}\right)}{\varphi\left(t_{n}\right)}=\beta_{n-1}
$$

It is important to point out that in order to calibrate the model, it is more convenient to consider a continuous function right $\widetilde{\varphi}$ such that $\widetilde{\varphi}\left(t_{i-1}\right)=1$, for $i=1, \ldots, n$. In this case, $\widetilde{\varphi}$ will not be continuous and instead of $\widetilde{\varphi}_{u}(u)$ in the pricing formula (12), we will have

$$
\prod_{i=1}^{n} \widetilde{\varphi}_{t_{i}}\left(t_{i}^{-}\right)
$$

where $\tilde{\varphi}\left(t^{-}\right)$stands for the left side limit. The pricing formula becomes less elegant but more efficient because, in this case, there is no need to solve the system of equations. We will be back to this approach later in section 5 .

\section{Options on zero-coupon bonds}

The price of an option over a bond, $h(P(t, u))$, may be written as:

$$
P(0, t) \cdot \frac{E\left(e^{-\int_{0}^{t} r(s) d s} h(P(t, u))\right)}{P(0, t)}=P(0, t) \cdot E_{t}^{*}(h(P(t, u))) .
$$


This is because $\exp \left\{-\int_{0}^{t} r(s) \mathrm{d} s\right\} / P(0, t)$ is a density function. The corresponding $P_{t}^{*}$ probability is called a $t$-forward martingale measure. Later on, we will find the $t$-forward martingale measure for the ECIR model. by

In the sequell, we will find the transition density of the process $U(t)$ defined

$$
U(t)=x+2 \int_{0}^{t} \sqrt{U(s)} \mathrm{d} B(s)+2 \int_{0}^{t}\left(F_{u}(s) U(s)+\frac{1}{2} \delta\right) \mathrm{d} s .
$$

The law of the process $U(t), t \leq u$ is the law of $X_{t}$ under the change of measure $Q_{x}^{\delta} \rightarrow Z_{u} Q_{x}^{\delta}$ where $Q_{x}^{\delta}$ is the law of $\mathrm{BESQ}^{\delta}$ and $\mathrm{Z}$ is defined as in formula (10). We will find the law of $U$ using the deterministic time change, and write $F=F_{u}$. First, set $\delta=1$. If $V(t)=v+W(t)+\int_{0}^{t} F(s) V(s) \mathrm{d} s$, then $U=V^{2}$ is the solution of

$$
U(t)=x+2 \int_{0}^{t} \sqrt{U(s)} \operatorname{sgn} V(s) \mathrm{d} W(s)+2 \int_{0}^{t} F(s) U(s) \mathrm{d} s+t, v=\sqrt{x},
$$

and $\int_{0}^{t} \operatorname{sgn} V(s) \mathrm{d} W_{s}$ is another Brownian motion. However, the equation

$$
V(t)=v+W_{t}+\int_{0}^{t} F(s) V(s) \mathrm{d} s
$$

is a well known linear equation and its solution is

$$
V(t)=\varphi(t)\left[v+\int_{0}^{t} \varphi^{-1}(s) \mathrm{d} W_{s}\right], \quad \frac{\varphi^{\prime}(s)}{\varphi(s)}=F(s), \quad \varphi(0)=1 .
$$

Since the stochastic integral of a deterministic function is Gaussian, we obtain that $V(t)$ is a Gaussian process with, for a given $t$, mean $v \varphi(t)$ and variance $\varsigma^{2}$, where

$$
\varsigma^{2}=\varsigma^{2}(t)=\varphi^{2}(t) \int_{0}^{t} \varphi^{-2}(s) \mathrm{d} s .
$$

Hence, still using $\delta=1$, the transition density of the process $U(t)$ is

$$
r_{t}^{1, \varphi}(x, \cdot)=q_{\varsigma^{2}}^{1}\left(x \varphi^{2}(t), \cdot\right)
$$

where $q^{1}$ is the transition density of $W_{t}^{2}$ (which is the squared Bessel process of dimension 1). Therefore $r_{t}^{\delta, \varphi}(x, \cdot)=q_{\tau^{2}}^{\delta}\left(x \varphi^{2}(t), \cdot\right)$, where $q^{\delta}$ is the transition density of the squared $\delta$-dimensional Bessel process. This fact results easily from the Pythagoras theorem (section 1.1) applied to the law of the process $U$. The proof is exactly the same as for BESQ processes.

Finally, we write well known formulas:

$$
q_{t}^{\delta}(x, y)=\frac{1}{2 t}\left(\frac{y}{x}\right)^{\nu / 2} \exp \left\{-\frac{(x+y)}{2 t}\right\} I_{\nu}\left(\frac{\sqrt{x y}}{t}\right),
$$


where $t>0, x>0$, and $I_{\nu}$ is the Bessel function of index $\nu=\delta / 2-1$, with

$$
I_{\nu}(x)=\left(\frac{x}{2}\right)^{\nu} \sum_{n=0}^{\infty} \frac{\left(\frac{x}{2}\right)^{2 n}}{n ! \Gamma(\nu+n+1)} .
$$

After these preliminaries, we will solve the problem of option pricing.

Applying the same method as in the preceding section we have, see formula (12), that

$$
P(\theta, T)=\exp \left\{\frac{1}{2} \delta\left[\ln \widetilde{\varphi}(T)-\int_{\theta}^{T} \beta_{s} d s\right]\right\} \exp \left\{\frac{1}{2} \cdot \frac{r_{\theta}}{\sigma_{\theta}}\left(-\beta_{\theta}+\tilde{\varphi}^{\prime}(\theta)\right)\right\} .
$$

In this case, we have that in $(\theta, T)$ the following relations hold:

$$
\frac{\widetilde{\varphi}^{\prime \prime}(s)}{\widetilde{\varphi}(s)}=h(s), \widetilde{\varphi}(\theta)=1, \frac{\widetilde{\varphi}^{\prime}(T)}{\widetilde{\varphi}(T)}=\beta_{T} .
$$

Therefore, in $[\theta, T], \widetilde{\varphi}(s)=\varphi_{T}(s) / \varphi_{T}(\theta)$. Now, $(P(\theta, T)-k)_{+}=(P(\theta, T)-k)$. $I\left[Y_{\theta}<k^{\prime}\right]$, where

$$
k^{\prime}=\frac{2\left\{\ln k+\frac{1}{2} \delta\left[\int_{\theta}^{T} \beta(s) \mathrm{d} s-\ln \varphi_{T}(T)+\ln \varphi_{T}(\theta)\right]\right\}}{\varphi_{T}^{\prime}(\theta) / \varphi_{T}(\theta)-\beta(\theta)},
$$

$k^{\prime}>0$, and $Y_{\theta}=r(\theta) / \sigma_{\theta}$. In the following propositions, for $u=\theta$, we will write $\psi$, and for $u=T$, we will write $\varphi$, instead of $\varphi_{\theta}$ and $\varphi_{T}$, respectively. Define two probabilities $P_{1}, P_{2}$ with densities related to the original probability law $P$ of the process $Y_{s}, s \leq \theta$ and $s \leq T$, respectively, as follows;

$$
\begin{aligned}
& \frac{\mathrm{d} P_{1}}{\mathrm{~d} P}=\frac{\exp \left(-\int_{0}^{\theta} Y_{s} \sigma_{s} \mathrm{~d} s\right)}{P(0, \theta)}, \\
& \frac{\mathrm{d} P_{2}}{\mathrm{~d} P}=\frac{\exp \left(-\int_{0}^{\theta} Y_{s} \sigma_{s} \mathrm{~d} s\right) P(\theta, T)}{P(0, T)} .
\end{aligned}
$$

Now, the following pricing formula holds.

Proposition 3.1. The price $C$ splits into two terms:

$$
C=P(0, T) \cdot P_{2}\left(\frac{r(\theta)}{\sigma_{\theta}}<k^{\prime}\right)-k P(0, \theta) \cdot P_{1}\left(\frac{r(\theta)}{\sigma_{\theta}}<k^{\prime}\right),
$$

where under the law $P_{1}, r(\theta) / \sigma_{\theta}$ has the density $r^{\delta}(x, \cdot)=q_{\varsigma^{2}}^{\delta}\left(x \psi^{2}(\theta), \cdot\right)$ with $x=r(0) / \sigma_{0}$, and $\varsigma^{2}=\psi^{2}(\theta) \cdot \int_{0}^{\theta}(\psi(s))^{-2} \mathrm{~d} s$, while in the analogous formula for the law $P_{2}$ we replace $\psi$ by $\varphi$. 
Proof:

$$
\begin{aligned}
& P_{1}\left(\frac{r(\theta)}{\sigma_{\theta}}<k^{\prime}\right)=P_{1}\left(Y_{\theta}<k^{\prime}\right) \\
& =\mathrm{E}\left[\frac{\exp \left\{-\int_{0}^{\theta} X_{s} \sigma_{s} \mathrm{~d} s \bar{Z}_{\theta} I\left[X_{\theta}<k^{\prime}\right]\right\}}{P(0, \theta)}\right] \\
& P_{2}\left(\frac{r(\theta)}{\sigma_{\theta}}<k^{\prime}\right)=P_{2}\left(Y_{\theta}<k^{\prime}\right) \\
& =\mathrm{E}\left[\frac{\exp \left\{-\int_{0}^{\theta} X_{s} \sigma_{s} \mathrm{~d} s P(\theta, T) \bar{Z}_{\theta} I\left[X_{\theta}<k^{\prime}\right]\right\}}{P(0, T)}\right]
\end{aligned}
$$

In the formula for $P(\theta, T)$, we clearly change $Y(\theta)=r(\theta) / \sigma(\theta)$ for $X(\theta)$. Simple algebra leads to

$$
P_{i}\left(Y_{\theta}<k^{\prime}\right)=E\left[Z_{i} I\left(X_{\theta}<k^{\prime}\right)\right],
$$

where $Z_{i}$ is an exponential martingale.

$$
Z_{i}=\exp \left\{\frac{1}{2}\left[-\delta \ln \gamma_{i}(\theta)+X_{\theta} \frac{\gamma_{i}^{\prime}(\theta)}{\gamma_{i}(\theta)}-\gamma_{i}^{\prime}(0) x-\int_{0}^{\theta} X_{s} h(s) \mathrm{d} s\right]\right\},
$$

$\gamma_{1}=\psi$, and $\gamma_{2}=\varphi$. Therefore $Z_{1}=Z_{\theta}$ in our previous notation. Generalizing the above results, we have the following proposition:

Proposition 3.2 The following relation holds:

$$
\mathrm{E}\left\{H(r(\theta)) \exp \left(-\int_{0}^{\theta} r(s) d s\right)\right\}=P(0, \theta) E_{\theta}^{*}(H(r(\theta))),
$$

where $\mathrm{E}_{\theta}^{*}$ is the expectation related to the probability distribution with density

$$
q_{\varsigma^{2}}^{\delta}\left(x ; \gamma^{2}(\theta), \cdot\right)
$$

Proof:

$$
\begin{aligned}
\mathrm{E}\left(H\left(Y_{\theta} \sigma_{\theta}\right) e^{-\int_{0}^{\theta} Y_{s} \sigma_{s} d_{s}}\right) & =\mathrm{E}\left\{H\left(X_{\theta} \sigma_{\theta}\right) \bar{Z}_{\theta} e^{-\int_{0}^{\theta} X_{s} \sigma_{s} d s}\right\} \\
& =P(0, \theta) E\left\{H\left(X_{\theta} \sigma_{\theta}\right) Z_{1}\right\} \\
& =P(0, \theta) E_{\theta}^{*}(H(r(\theta)))
\end{aligned}
$$

Here, $\mathrm{E}_{\theta}^{*}$ is precisely the expectation with respect to the $\theta$-forward martingale measure. This forward martingale measure was obtained here in a straightforward way; compare with Maghsoodi (1996). 


\section{Multifactor models}

The one-factor model is often criticized because that prices of bonds of different maturities are perfectly correlated. Prices are deterministic functions of $r(t)$ if we valuate bonds at time $t$. Pricing interest rate derivatives with multifactor models in the case of independent factors does not present additional analytical difficulties, but clearly numerical calculations will be more complicated.

We will consider the two-factor model, and begin with independent factors. Let $r(t)=r_{1}(t) \oplus r_{2}(t)$. As in the section 1, the operator $\Theta$ means the sum of independent terms. The process $r(t)$ becomes a one-factor model if and only if

$$
\sigma_{1}(t)=\sigma_{2}(t), \beta_{1}(t)=\beta_{2}(t) .
$$

Otherwise, the general valuation formula has the form

$$
\begin{aligned}
C & =\mathrm{E}\left[e^{-\int_{0}^{\theta} r(s) \mathrm{d} s} H\left(r_{1}(\theta), r_{2}(\theta)\right)\right] \\
& =P(0, \theta) \cdot \mathrm{E}_{\theta}^{*}\left[H\left(r_{1}(\theta), r_{2}(\theta)\right)\right],
\end{aligned}
$$

where $\mathrm{E}_{\theta}^{*}$ is the expectation related to the modified independent process. Through the usual conditioning technique, the pricing problem might be reduced to univariate integrations.

The special structure of the CIR model, and particularly the Shiga \& Watanabe theorem, allows a relatively easy approach, even in the case of dependent factors. The general multifactor ECIR model is defined in Rogers (1995). We propose, here, an alternative approach which allows for dependency of factors measured by corresponding correlations.

We will show here the general procedure of reducing the problem in our setting to independent factors with the sole application to pricing zero-coupon bonds. The term "factor" will be slightly more general than before. Let

$$
Y_{i}(t)=2 \sqrt{Y_{i}(t)} \mathrm{d} W_{i}(t)+\left(2 \beta_{i}(t) Y_{i}(t)+\delta_{i}\right) \mathrm{d} t .
$$

An easy application of the Shiga \& Watanabe theorem shows that $Y_{i}(t)$ might be decomposed into the sum of independent processes $Y_{i}^{1}(t)$ and $Y_{i}^{2}(t)$ with independent underlying Brownian motions $W_{i}^{(1)}$ and $W_{i}^{(2)}$. We will assume that $W_{1}^{(2)}$ and $W_{2}^{(2)}$ are independent, and the dependency between $r_{1}(t)$ and $r_{2}(t)$ will result from the dependency between $Y_{1}^{1}$ and $Y_{2}^{1}$, both driven by $W=W_{1}^{(1)}=W_{2}^{(1)}$. The bond price $P(0, T)$ becomes:

$$
\mathrm{E}\left[e^{-\int_{0}^{T}\left(\sigma_{1}(s) Y_{1}^{-1}(s)+\sigma_{2}(s) Y_{2}^{1}(s)\right) \mathrm{d} s}\right] \mathrm{E}\left[e^{-\int_{0}^{T} \sigma_{1}(s) Y_{1}^{2}(s) \mathrm{d} s}\right] \mathrm{E}\left[e^{-\int_{0}^{T} \sigma_{2}(s) Y_{2}^{2}(s) \mathrm{d} s}\right] .
$$


The analytical method for the calculation of the first term is based on the space-time transformation of a squared Bessel process. The method might be performed in a more general setting, but our presentation (for simplicity) will only consider $r_{1}^{1}$ and $r_{2}^{1}$, with constant parameters $\beta_{1}, \beta_{2}<0$. In what follows, we will assume $\delta_{1}^{(1)}=\delta_{2}^{(1)}=\delta$. In this case, we set

$$
Y_{i}^{1}(t)=e^{2 \beta_{i} t} X\left(\frac{1-e^{-\beta_{i} t}}{2 \beta_{i}}\right)
$$

where $X$ is the $\delta$-dimensional squared Bessel process (see the beginning of section 3 ). In this case,

$$
\int_{0}^{t} \sigma_{1}(s) Y_{1}^{1}(s) d s=\int_{0}^{t} \sigma_{1}(s) e^{2 \beta_{1} s} X\left(\frac{1-e^{-2 \beta_{1} s}}{2 \beta_{1}}\right) \mathrm{d} s .
$$

The deterministic function

$$
C(t)=\frac{1-e^{-2 \beta_{1} t}}{2 \beta_{1}}
$$

is a very simple time change with $C(0)=0$. Notice now that

$$
\int_{0}^{t} \sigma_{1}(s) Y_{1}^{1}(s) \mathrm{d} s=\int_{0}^{C(t)}\left(1-2 \beta_{1} u\right)^{-2} \sigma_{1}\left[\frac{\ln \left(1-2 \beta_{1} u\right)}{-2 \beta_{1}}\right] X(u) \mathrm{d} u .
$$

Therefore, the first term in (21) might be calculated as in section 1 . obtain

Furthermore, assuming $\beta_{2}<\beta_{1}<0$, after straightforward calculations, we

$$
\operatorname{Cov}\left(r_{1}(t), r_{2}(t)\right)=2 \delta \sigma_{1}(t) \sigma_{2}(t) e^{2\left(\beta_{1}+\beta_{2}\right) t}\left(\frac{1-e^{-2 \beta_{1} t}}{2 \beta_{1}}\right)^{2}
$$

This approach, if ever put into practice, requires the identification of factors as some observable variables. We simply consider this possibility as it avoids solutions of multidimensional Riccati equations, $c f$. Rogers (1995).

\section{Calibration of the model}

We remind from the section 2 that the price of the bond $P(0, t)$ is in our model

$$
P(0, t)=\exp \frac{1}{2}\left\{\delta\left[\ln \prod_{i=1}^{n} \tilde{\varphi}\left(t_{i}^{-}\right)-\int_{0}^{t} \beta_{s} \mathrm{~d} s\right]+x\left[-\beta_{0}+\tilde{\varphi}(0)\right]\right\}
$$

where $x=r_{0} / \sigma_{0}$. 


\subsection{The proposed method}

We choose the parameter $\delta$ fixed and allow changes in $\beta$ and $\sigma$ every week. More precisely, at any time $t$, say a fixed day of a week, we observe prices of bonds at different maturities:

$$
O(i, i+j), \text { for } i=1, \ldots, n, \text { and } j=1, \ldots, d .
$$

For any choice of parameters we recover $r_{i}^{(j)}$. Usually $r_{i}^{(j)} \neq r_{i}^{(k)}$ for $k \neq j$. This occurs because we consider a one-dimensional (one-factor) model of a $d$ dimensional problem.

We set $r_{i}=\frac{1}{d} \sum_{j=1}^{d} r_{i}^{(j)}$ as a theoretical value of the process at time $t_{i}$. With this $r_{i}$ (and the same parameters), we choose $P(i, i+j)$ as a theoretical value of the corresponding bond. Our goal is to choose the path route of parameters that minimize:

$$
\sum_{i=1}^{n} \sum_{j=1}^{d}[P(i, i+j)-O(i, i+j)]^{2} .
$$

To solve the problem, we use a fundamental gradient descent method, known as steepest descent. The basic idea is to move along the negative direction of the objective function.

\subsection{Illustrative examples}

To show how our method works in practice, we consider the prices of the Mexican treasury certificates (CETES) and the prices of the American treasury bills (T-bills) for 91, 182 and 365 days (or 13,26 and 52 weeks). Data considers 130 weeks from September $11^{\text {th }}, 1997$ to March $2^{\text {nd }}, 2000$. As an initial point to the problem, we take the CIR model parameters, obtained by a traditional method, for each of the financial instruments (CETES and T-bills).

\subsubsection{CETES}

In this case, the initial (CIR) parameter values are:

$$
\begin{aligned}
& \delta=2.24228790, \\
& \beta=-0.91145494, \\
& \sigma=0.35791831 .
\end{aligned}
$$

We got the following results: the objective function value is reduced 3.7 times, from 0.00443073 (CIR) to 0.00120477 (ECIR). The CETES bond prices adjustment performance is showed at table 5.1. 
Table 5.1 CETES bond prices adjustment performance.

\begin{tabular}{|c|c|c|c|}
\hline & CIR $\sum \varepsilon^{2}$ & ECIR $\sum \varepsilon^{2}$ & CIR $\sum \varepsilon^{2} /$ ECIR $\sum \varepsilon^{2}$ \\
\hline 13 Weeks & 0.00070426 & 0.00028588 & 2.5 \\
\hline 26 Weeks & 0.00043253 & 0.00040726 & 1.1 \\
\hline 52 Weeks & 0.00329394 & 0.00051163 & 6.4 \\
\hline Total & 0.00443073 & 0.00120477 & 3.7 \\
\hline
\end{tabular}

\subsubsection{T-bills}

In this case, the initial (CIR) parameter values are:

$$
\begin{aligned}
& \delta=1.42056719 \\
& \beta=-0.53749523, \\
& \sigma=0.11286799
\end{aligned}
$$

After 10 iterations, we got the following result: the objective function value is reduced 20.2 times, from 0.00084535 (CIR) to 0.00004186 (ECIR). The T-bills bond prices adjustment performance is showed at table 5.2.

Table 5.2. T-bills bond prices adjustment performance.

\begin{tabular}{|c|c|c|c|}
\hline & CIR $\sum \varepsilon^{2}$ & ECIR $\sum \varepsilon^{2}$ & CIR $\sum \varepsilon^{2} /$ ECIR $\sum \varepsilon^{2}$ \\
\hline 13 Weeks & 0.00003637 & 0.00000463 & 7.9 \\
\hline 26 Weeks & 0.00003714 & 0.00001661 & 2.2 \\
\hline 52 Weeks & 0.00077185 & 0.00002062 & 37.4 \\
\hline Total & 0.00084535 & 0.00004186 & 20.2 \\
\hline
\end{tabular}

\section{Conclusions and final remarks}

We have proposed an alternative approach to the extended CIR Model, taking a squared Bessel process as the basic one. We have shown that valuation and calibration are easier and more transparent when using our parametrization. The past and present history of prices affect future parameter values (functions of time). Needless to say, more studies are needed to see if our historical approach can help for further forecasting. It is important to point out that our method of calibrations can be extended to other interest rate derivatives and to multifactor models, even in the case of dependent factors as in our proposed method. 


\section{References}

Cox, J. C., J. E. Ingersoll, and S. A. Ross, (1985). A Theory of Term Structure of Interest Rates. Econometrica, 53, pp. 385-408.

Hull, J., and A. White, (1990). Pricing interest rate derivative securities, Review of Financial Studies, Vol. 3, pp. 573-592.

Maghsoodi, Y., (1996), Solution of the Extended CIR Term Structure and Bond Option Valuation, Mathematical Finance, Vol. 6, pp. 89-109.

Revuz, D., and M. Yor, (1994), Continuous Martingales and Brownian Motion, SpringerVerlag.

Rogers, L. C. G., (1995), Which Model for Term-Structure of Interest Rates Should One Use?, Mathematical Finance, The IMA Volumes in Mathematics and Its Applications, Vol. 65. Springer-Verlag. pp. 93-115.

Schlögl, E., and L. Schlögl, (1997), A Tractable Term Structure Model with Endogenous Interpolation and Positive Interest Rates, Preprint, Department of Statistics, University of Bonn.

Yor, M., (1992), Some Aspects of Brownian Motion, Part I: Some Special Functionals, Birkhäuser Verlag.

Vasicek, O., (1977), An Equilibrium Characterization of the Term Structure, Journal of Financial Economics, Vol. 5, pp. 177-188. 